\title{
Genetic programming method for modelling of cup height in deep drawing process
}

\author{
Gusel, L. ${ }^{a}{ }^{,}$, Boskovic, V. ${ }^{b}$, Domitner, J. ${ }^{b}$, Ficko, M. ${ }^{a}$, Brezocnik, M. ${ }^{a}$ \\ aUniversity of Maribor, Faculty of Mechanical Engineering, Maribor, Slovenia \\ ${ }^{\mathrm{b}}$ Graz University of Technology, Institute of Materials Science, Joining and Forming, Graz, Austria
}

\section{A B S T R A C T}

Genetic programming method for modelling of maximum height of deep drawn high strength sheet materials is proposed in this paper. Genetic programming (GP) is an evolutionary computation approach which uses the principles of Darwin's natural selection to develop effective solutions for different problems. The aim of the research was the modelling of cylindrical cup height in deep drawing process and analysis of the impact of process parameters on material formability. High strength steel sheet materials (DP1180HD and DP780) were formed by deep drawing using different punch speeds and blank holder forces. The heights of specimens before cracks occur were measured. Therefore, four input parameters (yield stress, tensile strength, blank holder force, punch speed) and one output parameter (cup height) were used in the research. The experimental data were the basis for obtaining various accurate prediction models for the cup heights by the genetic programming method. Results showed that proposed genetic modelling method can successfully predict fracture problems in a process of deep drawing.
\end{abstract}

\author{
ARTICLE INFO \\ Keywords: \\ Metal forming; \\ Deep drawing; \\ Modelling; \\ Genetic programming \\ *Corresponding author: \\ leo.gusel@um.si \\ (Gusel, L.) \\ Article history: \\ Received 19 December 2017 \\ Revised 22 February 2018 \\ Accepted 7 June 2018
}

\section{References}

[1] Koza, J. (1992). Genetic programming, The MIT Press, Massachusetts, USA.

[2] Pawlak, T.P. (2015). Competent algorithms for geometric semantic genetic programming, PhD thesis, University of Technology, Poznan, Poland, doi: 10.13140/RG.2.1.1240.7760.

[3] Kakandikar, G.M., Nandedkar, V.M. (2016). Prediction and optimization of thinning in automotive sealing cover using genetic algorithm, Journal of Computational Design and Engineering, Vol. 3, No. 1, 63-70, doi: 10.1016/j. jcde.2015.08.001.

[4] Gharib, H., Wifi, A.S., Younan, M., Nassef, A. (2006). Optimization of the blank holder force in cup drawing, Journal of Achievements in Materials Manufacturing Engineering, Vol. 18, No. 1-2, 291-294.

[5] Sener, B., Kurtaran, H. (2016). Optimization of process parameters for rectangular cup deep drawing by the Taguchi method and genetic algorithm, Materials Testing, Vol. 58, No. 3, 238-245, doi: 10.3139/120.110840.

[6] Wei, L., Yuying, Y. (2008). Multi-objective optimization of sheet metal forming process using Pareto-based genetic algorithm, Journal of Materials Processing Technology, Vol. 208, No. 1-3, 499-506, doi: 10.1016/i.jmatprotec. 2008.01.014.

[7] Di Lorenzo, R., Ingarao, G., Marretta, L., Micari, F. (2009). Deep drawing process design: A multi objective optimization approach, Key Engineering Materials, Vol. 410-411, 601-608, doi: 10.4028/www.scientific.net/KEM.410$\underline{411.601 .}$

[8] Naceur, H., Guo, Y.Q., Batoz, J.L. (2004). Blank optimization in sheet metal forming using an evolutionary algorithm, Journal of Materials Processing Technology, Vol. 151, No. 1-3, 183-191, doi: 10.1016/i.jmatprotec.2004. $\underline{04.036 .}$.

[9] Singh, D., Yousefi, R., Boroushaki, M. (2011). Identification of optimum parameters of deep drawing of a cylindrical workpiece using neural network and genetic algorithm, International Journal of Mechanical, Aerospace, Industrial, Mechatronic and Manufacturing Engineering, Vol. 5, No. 6, 987-993, doi: 10.1999/1307-6892/2043. 
[10] Zhao, J., Wang, F. (2005). Parameter identification by neural network for intelligent deep drawing of axisymmetric workpieces, Journal of Materials Processing Technology, Vol. 166, No. 3, 387-391, doi: 10.1016/j.jmatprotec. 2004.08.020.

[11] Tinkir, M., Dilmeç, M., Türköz, M., Halkaci, H.S. (2015). Investigation of the effect of hydromechanical deep drawing process parameters on formability of AA5754 sheets metals by using neuro-fuzzy forecasting approach, Journal of Intelligent \& Fuzzy Systems, Vol. 28, No. 2, 647-659, doi: 10.3233/IFS-141346.

[12] Öztürk, E., Türköz, M., Halkacı, H.S., Koç, M. (2017). Determination of optimal loading profiles in hydromechanical deep drawing process using integrated adaptive finite element analysis and fuzzy control approach, The International Journal of Advanced Manufacturing Technology, Vol. 88, No. 9-12, 2443-2459, doi: 10.1007/ s00170-016-8912-x.

[13] Oduguwa, V., Tiwari, A., Roy, R. (2005). Evolutionary computing in manufacturing industry: An overview of recent applications, Applied Soft Computing, Vol. 5, No. 3, 281-299, doi:10.1016/j.asoc.2004.08.003.

[14] Brezocnik, M., Kovacic, M., Gusel, L. (2005). Comparison between genetic algorithm and genetic programming approach for modeling the stress distribution, Materials and Manufacturing Processes, Vol. 20, No. 3, 497-508, doi:10.1081/AMP-200053541.

[15] Gusel, L., Rudolf, R., Brezocnik, M. (2015). Genetic based approach to predicting the elongation of drawn alloy, International Journal of Simulation Modelling, Vol. 14, No. 1, 39-47, doi: 10.2507/IJSIMM14(1)4.277.

[16] Halder, C., Madej, L., Pietrzyk, M., Chakraborti, N. (2015). Optimization of cellular automata model for the heating of dual-phase steel by genetic algorithm and genetic programming, Materials and Manufacturing Processes, Vol. 30, No. 4, 552-562, doi: 10.1080/10426914.2014.994765.

[17] Kovacic, M., Uratnik, P., Brezocnik, M., Turk, R. (2007). Prediction of the bending capability of rolled metal sheet by genetic programming, Materials and Manufacturing Processes, Vol. 22, No. 5, 634-640, doi: $10.1080 / 10426910701323326$.

[18] Radakovics, S.M. (2017). Einfluss der Tiefziehgeschwindigkeit auf die Kaltumformbarkeit von HSS und AHSS Stählen, Bachelor's thesis, Fakultät für Maschinenbau und Wirtschaftswissenschaften, TU Graz, Austria (in German). 
APEM
Advances in Production Engineering \& Management Letnik 13 | Številka 3 | September 2018 | Strani 358-365 https://doi.org/10.14743/apem2018.3.296
ISSN 1854-6250

Spletna stran: apem-journal.org Izvirni znanstveni članek

\title{
Modeliranje višine skodelice pri globokem vleku z metodo genetskega programiranja
}

\author{
Gusel, L. ${ }^{a,}{ }^{,}$, Boskovic, V. ${ }^{b}$, Domitner, J. ${ }^{b}$, Ficko, M. ${ }^{a}$, Brezocnik, M. $^{\mathrm{a}}$ \\ ${ }^{a}$ University of Maribor, Faculty of Mechanical Engineering, Maribor, Slovenia \\ ${ }^{\mathrm{b}}$ Graz University of Technology, Institute of Materials Science, Joining and Forming, Graz, Austria
}

\section{POVZETEK}

$\mathrm{V}$ prispevku je predlagana metoda genetskega programiranja za modeliranje maksimalne višine globoko vlečenih materialov visoke trdnosti. Genetsko programiranje (GP) je evolucijski računski postopek, ki uporablja načela darvinistične naravne selekcije za razvoj učinkovitih rešitev za različne probleme. Cilj raziskave je bil modeliranje višine cilindrične skodelice z globokim vlekom in analiza vpliva procesnih parametrov na sposobnost materiala za preoblikovanje. $\mathrm{Z}$ globokim vlekom smo preoblikovali dve vrsti jeklene pločevine $\mathrm{z}$ visoko trdnostjo (DP1180HD in DP780) z uporabo različnih hitrosti preoblikovanja in vpenjalnih sil vpenjalne naprave. Izmerili smo višine vzorcev pred pojavom razpok. V raziskavi so bili uporabljeni štirje vhodni parametri (napetost tečenja, natezna trdnost, vpenjalna sila, udarna hitrost) in en izhodni parameter (višina skodelice). Eksperimentalni podatki so bili osnova za pridobitev različno uspešnih napovedovalnih modelov za višino skodelic z metodo genskega programiranja. Rezultati so pokazali, da predlagana metoda genetskega programiranja uspešno napoveduje težave povezane $\mathrm{z}$ lomom pri postopku globokega vleka.

\section{PODATKI O ČLANKU}

Ključne besede:

Preoblikovanje kovin;

Globoki vlek;

Modeliranje;

Genetsko programiranje;

*Kontaktna oseba:

leo.gusel@um.si

(Gusel, L.)

Zgodovina članka:

Prejet 19. decembra 2017

Popravljen 22. februarja 2018

Sprejet 7.junija 2018 